\title{
The Role of Size and Charge for Blood-Brain Barrier Permeation of Drugs and Fatty Acids
}

\author{
Anna Seelig
}

Published online: 27 June 2007

(C) Humana Press Inc. 2007

\begin{abstract}
The lipid bilayer is the diffusion barrier of biological membranes. Highly protective membranes such as the bloodbrain barrier (BBB) are reinforced by $\mathrm{ABC}$ transporters such as $P$-glycoprotein (MDR1, ABCB1) and multidrug resistance associated proteins (MRPs, ABCCs). The transporters bind their substrates in the cytosolic lipid bilayer leaflet before they reach the cytosol and flip them to the outer leaflet. The large majority of drugs targeted to the central nervous system (CNS) are intrinsic substrates of these transporters. Whether an intrinsic substrate can cross the BBB depends on whether passive influx is higher than active efflux. In this paper, we show that passive influx can be estimated quantitatively on the basis of Stokesian diffusion, taking into account the ionization constant and the cross-sectional area of the molecule in its membrane bond conformation, as well as the lateral packing density of the membrane. Active efflux by ABC transporters was measured. The calculated net flux is in excellent agreement with experimental results. The approach is exemplified with several drugs and fatty acid analogs. It shows that compounds with small cross-sectional areas $\left(A_{\mathrm{D}}<70 \AA^{2}\right)$ and/ or intermediate or low charge exhibit higher passive influx than efflux and, therefore, cross the BBB despite being intrinsic substrates. Large $\left(A_{\mathrm{D}}>70 \AA^{2}\right)$ or highly charged compounds show higher efflux than influx. They cannot cross the $\mathrm{BBB}$ and are, thus, apparent substrates for $\mathrm{ABC}$ transporters. The strict size and charge limitation for $\mathrm{BBB}$ permeation results from the synergistic interaction between passive influx and active efflux.
\end{abstract}

A. Seelig $(\bowtie)$

Biophysical Chemistry, Biozentrum, University of Basel,

Klingelbergstrasse 70,

Basel, Switzerland

e-mail: Anna.Seelig@unibas.ch
Keywords $P$-glycoprotein $\cdot$ Passive influx $\cdot$ Stokes · Active efflux $\cdot$ Membrane packing density

\section{Introduction}

The limitation of intestinal barrier (IB) and blood-brain barrier (BBB) permeation by the size and the charge of molecules has long been observed (Didziapetris et al. 2003; Fischer et al. 1998; Fischer et al. 1998; Gerebtzoff and Seelig 2006; Golin et al. 2003; Pauletti et al. 1997; Varma et al. 2005). For an understanding at the molecular level, the lipid bilayer, the efflux transporters, and the permeating drugs have to be characterized (Seelig and Gatlik-Landwojtowicz 2005). Most drugs reach the central nervous system (CNS) by passive diffusion across the BBB. The BBB consists of the endothelial cells of the blood capillaries in the brain that have tight junctions that prevent paracellular diffusion. Molecules targeted to the CNS, thus, have to cross the lipid bilayer of the endothelial cell membrane. The barrier property of the bilayer varies with the lipid composition and the physical state of the lipids. Under physiological conditions, the lipid bilayer is in the liquid crystalline state and behaves like an optically uniaxial crystal with the optical axis perpendicular to the surface of the bilayer. Lipid bilayers are, thus, anisotropic systems in marked contrast to isotropic organic solvents (e.g., octanol or hexadecane) often used as membrane-mimicking systems.

Using solid state nuclear magnetic resonance (NMR) techniques, a quantitative analysis of the molecular ordering and dynamics of lipid bilayers became possible with a segmentto-segment resolution. Well-defined conformations were observed for the glycerol backbone and, to some extent, also for the polar head groups. The packing of the fatty acyl chains is best described in terms of a statistical order profile. For a given 
membrane, the average order of the fatty acyl chains is comparatively high, close to the head group region and decreases toward the center of the membrane due to increasing cis-trans isomerization of the $\mathrm{C}-\mathrm{C}$ bonds (for reviews, see Seelig 2002; Seelig 1980). The overall membrane order increases with increasing percentage of lipids with small polar head groups, such as phosphatidyethanolamine, sphingomyelin, or cholesterol, and decreases with increasing percentage of lipids with unsaturated fatty acyl chains or with increasing concentration of amphiphilic guest molecules (e.g., drugs; Meier et al. 2006). Transmembrane proteins perfectly match the lipid bilayer properties and barely influence the lipid order (for reviews, see Seelig 2002; Seelig 1980).

A further possibility to characterize the properties of lipid bilayer membranes is to assess their lateral lipid packing density $\left(\pi_{\mathrm{M}}\right)$ in comparison to the lateral packing density or surface pressure of a lipid monolayer (Seelig 1987). Biological membranes exhibit a range of lateral packing densities from $\pi_{\mathrm{M}} \approx 28 \mathrm{mN} / \mathrm{m}$ for the IB, to $\pi_{\mathrm{M}} \approx$ $35 \mathrm{mN} / \mathrm{m}$ for the BBB, and $\pi_{\mathrm{M}} \approx 45 \mathrm{mN} / \mathrm{m}$ for membranes with a high content of phosphatidylethanolamines (GatlikLandwojtowicz et al. 2006; Seelig 2006).

Using lipid monolayers as membrane model systems revealed that the energy $(\Delta W)$ required for membrane insertion is proportional to the lateral packing density of the lipid layer $\left(\pi_{\mathrm{M}}\right)$ and the cross-sectional area $\left(A_{\mathrm{D}}\right)$ of the penetrating compound $\left(\Delta W=A_{\mathrm{D}} \pi_{\mathrm{M}}\right.$; Boguslavsky et al. 1994). The lipid-water partition coefficient, therefore, decreases exponentially with increasing membrane packing density $\left(\pi_{\mathrm{M}}\right)$ and increasing cross-sectional area $\left(A_{\mathrm{D}}\right)$ of the molecule (Boguslavsky et al. 1994; Fischer et al. 1998; Gerebtzoff et al. 2004). As membrane insertion is the first step in the process of passive diffusion, it also depends on the two parameters $\left(\pi_{\mathrm{M}}\right)$ and $\left(A_{\mathrm{D}}\right)$ (Seelig and GatlikLandwojtowicz 2005).

Molecules spanning half the lipid bilayer such as longchain fatty acids or lipid molecules reach the other side of the membrane by a flip-flop mechanism rather than by diffusion (for review, see Hamilton 1998; Hamilton and Kamp 1999). However, like diffusion, flip-flop also depends on the cross-sectional area of the compound and the lateral packing density of the membrane as will be discussed in more detail below.

The lipid bilayer is not only a barrier for large but also for charged molecules. Although, molecules insert into the membrane interface in their charged form, they can cross the hydrophobic core of the membrane only in their uncharged form (Kamp and Hamilton 1992; Saparov et al. 2006). A rare exception is, e.g., the tetraphenylphosphonium ion that exhibits a highly delocalized charge (Altenbach and Seelig 1985).

A calibration diagram for BBB permeation established with 53 drugs with clinically well-tested ability to reach or not to reach the CNS (Fischer et al. 1998) revealed a limiting cross-sectional area of $A_{\mathrm{D}}=73 \AA^{2}$ (rounded to $A_{\mathrm{D}} \approx$ $80 \AA^{2}$ ) and a limiting ionization constant of $\mathrm{p} K_{\mathrm{a}} \approx 10$ and $\mathrm{p} K_{\mathrm{a}} \approx 4$ for bases and acids, respectively. We recently investigated a new set of compounds for BBB permeation using an in silico procedure to calculate the cross-sectional area of molecules in their membrane-bound conformation (Gerebtzoff and Seelig 2006). This new approach revealed a similar limiting cross-sectional area of $A_{\mathrm{D}} \approx 70 \AA^{2}$ for BBB permeation. An analogous calibration for the IB yielded a somewhat larger cut-off area of $A_{\mathrm{D}} \approx 100 \AA^{2}$, a similar limiting ionization constant for bases and a lower limiting ionization constant for acids due to the low $\mathrm{pH}$ in the stomach (Fischer et al. 1998). The strict area and charge limitation observed in the calibration diagrams cannot be fully understood on the basis of the exponential area dependence of membrane insertion (Boguslavsky et al. 1994; Fischer et al. 1998).

The barrier properties of membranes with protective functions such as the $\mathrm{BBB}$ are reinforced by $\mathrm{ABC}$ transporters including $P$-glycoprotein (Pgp, $\mathrm{ABCB} 1$ ), the recently identified breast cancer resistance protein (BCRP, ABCG2), and several members of the multidrug resistance-associated protein (MRPs, ABCCs) family (for review, see e.g., Begley 2004). Pgp is located in the apical membrane of the endothelial cells. Its expression level varies with genetic predisposition, age, food, and medication (Seelig and GatlikLandwojtowicz 2005 and references therein). It reduces the influx of a large variety of cationic or electrically neutral, exogenous drugs and toxins into the CNS by binding them in the inner, cytosolic leaflet of the lipid bilayer before they reach the cytosol, and flipping them to the outer leaflet or directly back into the blood using the energy of ATP hydrolysis. If Pgp is absent as, e.g., in collies (Mealey et al. 2001) or knock-out mice (Schinkel et al. 1995), the CNS concentration of certain drugs or toxins are much higher than in individuals protected by Pgp. MRPs export negatively charged and electrically neural compounds. The two efflux proteins, Pgp and MRP1, recognize their substrates via characteristic hydrogen bond acceptor patterns and share electrically neutral substrates (Gatlik-Landwojtowicz et al. 2006; Seelig 1998; Seelig et al. 2000).

Net flux across a cell layer is generally assessed by comparing basolateral-to-apical with apical-to-basolateral transport in confluent cell monolayers (Burton et al. 2002). The apparent or net transport $(J)$ across the BBB is the sum of passive influx $(\phi)$ into the cytosol, active export $(-V)$ by $\mathrm{ABC}$ transporters from the apical membrane into the apical medium, and passive bidirectional diffusion across the basolateral membrane. Under the assumption that the passive diffusion across the basolateral membrane is identical in both directions, the net influx $(J)$ across a cell membrane can be estimated as the sum of passive influx 


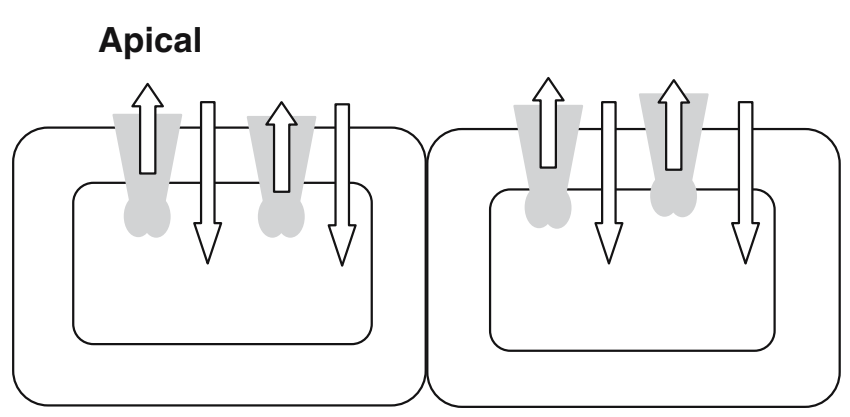

\section{Basolateral}

Figure 1 Cartoon of the endothelial cells that constitute the BBB. Most drugs reach the cytosol or cross the cell layer by passive diffusion (influx, $\Phi$, is indicated by an arrow into cytosol). The apical membrane harbors ATP transporters such as Pgp that efflux compounds out of the apical membrane before they reach the cytosol (efflux, $-V$, is indicated by an arrow out of the cell). The transport across the basolateral membrane is assumed to be identical in both directions (not indicated)

$(\Phi)$ and active efflux (- $V)$ across the apical membrane (Seelig and Gatlik-Landwojtowicz 2005; Seelig 2006) (see Fig. 1). We will show that net flux is membrane specific and concentration dependent.

The aim of the present investigation was to deconvolve passive influx $(\Phi)$ across the lipid bilayer and active efflux $(-V)$ by $\mathrm{ABC}$ transporters, and to assess the role of size (Seelig and Gatlik-Landwojtowicz 2005) and charge for the two processes. Passive influx was calculated on the basis of Stokesian diffusion, taking into account the lateral packing density of the membrane (Gatlik-Landwojtowiczet et al. 2006), as well as the charge and the cross-sectional area of the compound in its membrane-bound conformation. The latter was determined either by surface activity measurements (Fischer et al. 1998) or by molecular modeling (Gerebtzoff and Seelig 2006). Active efflux (-V) was measured in MDR1-transfected mouse embryo fibroblasts, exhibiting a similar expression level of $A B C$ transporters as the BBB. The concept is illustrated with 12 drugs that are known to be intrinsic (although not necessarily apparent) substrates for Pgp (Gatlik-Landwojtowicz et al. 2006), and 3 fatty acids that, in analogy to valproic acid (Ogawa et al. 2006) can be assumed to be intrinsic (although not necessarily apparent) substrates for MRPs.

The analysis reveals that intrinsic substrates with small cross-sectional areas $\left(A_{\mathrm{D}}<70 \AA^{2}\right)$ and low charge show much higher passive influx than efflux and, therefore, easily cross the $\mathrm{BBB}$, whereas intrinsic substrates with larger cross-sectional areas $\left(A_{\mathrm{D}}>70 \AA^{2}\right)$ and/or high charge show lower influx than efflux and cannot reach the CNS. The latter compounds are, thus, also apparent substrates at the BBB.

\section{Materials and Methods}

\section{Concept of Passive Diffusion}

The diffusion of small hydrophobic non-electrolytes such as benzene is too fast to be measured but was investigated by molecular dynamics simulations (Bassolino-Klimas et al. 1993). After insertion into the well-ordered, highly packed interfacial lipid region of the membrane (which was the ratelimiting step), benzene was shown to move from one packing defect to the next, whereby the rate of diffusion increased with increasing cis-trans isomerization of the fatty acyl chains toward the center of the membrane. The diffusion of small hydrophilic compounds (e.g., acetamide or urea) can in contrast be measured and was well described by simple Stokesian diffusion (Finkelstein 1987). For a given membrane, the permeability coefficients correlated linearly with hexadecane-water partition coefficients. However, for membranes of different lipid composition, permeability coefficients varied up to two orders of magnitude, decreasing with increasing membrane packing density. These observations show that the isotropic organic solvent hexadecane cannot account for the lateral packing densities of the different lipid membranes. A comparison of the permeability coefficients of linear and branched compounds in a given membrane suggested that the cross-sectional area rather than the molecular weight of the molecule is relevant for diffusion.

Under physiological conditions, the diffusion of most amphiphilic drugs is also too fast to be measured. In this study, we calculate influx on the basis of Stokesian diffusion, taking into account the cross-sectional area of the drug in its membrane-bound conformation, as well as the lateral packing density of the membrane (Seelig and Gatlik-Landwojtowicz 2005).

\section{Calculation of Passive Influx on the Basis of Stokesian Diffusion Taking Into Account the Lateral Membrane Packing Density}

The permeability coefficient $(P)$ is proportional to the product of the lipid-water partition coefficient $\left(\gamma_{\mathrm{lw}}\right)$ and the diffusion coefficient $(D)$ of the drug and inversely proportional to the thickness of the membrane $(\Delta x)$

$P=\gamma_{1 \mathrm{w}} \cdot D / \Delta x$

The lipid-water partition coefficient $\left(\gamma_{\text {lw }}\right)$ used in the context of membrane permeation is generally dimensionless and is defined as

$\gamma_{1 w}=C_{\mathrm{m}} / C_{\mathrm{eq}}$,

where $C_{\mathrm{m}}$ is the membrane and $C_{\mathrm{eq}}$ the aqueous concentration. The diffusion coefficient $(D)$ is inversely related to the 
radius $(r)$ of the diffusing particle and the viscosity of the membrane $(\eta)$,

$D=k T /(6 \pi \eta r)$.

The flux $(\Phi)$ is then defined as the product of the permeability coefficient ( $P$; Eq. 1$)$ and the concentration gradient between the intracellular and extracellular environment $(\Delta C)$. If only the initial external aqueous concentration $(C)$ is considered, the flux can be expressed as

$\Phi=C \cdot P$

To take into account the lateral packing density of the lipid membrane $\left(\pi_{\mathrm{M}}\right)$ and the cross-sectional area of the drug in its amphiphilic orientation $\left(A_{\mathrm{D}}\right)$, we derived the lipidwater partition coefficient $\left(K_{1 w}\right)$ from the measured air-water partition coefficient $\left(K_{\mathrm{aw}}\right)$ as

$K_{\mathrm{lw}}=K_{\mathrm{aw}} \cdot e^{-\pi_{\mathrm{M}} A_{\mathrm{D}} / \mathrm{kT}}$,

where kT is the thermal energy (Fischer et al. 1998). The dimensionless lipid-water partition coefficient $\left(\gamma_{l_{\mathrm{w}}}\right)$ is then transformed to the lipid-water partition coefficient $\left(K_{\mathrm{lw}}\right)$ with the dimension $\left[M^{-1}\right]$,

$\gamma_{1 w}=b \cdot K_{1 w},$.

where $b$ is the correction factor with the dimension [mol/l]. If we assume for simplicity that one mole of lipid corresponds approximately to 11 of lipid, the correction factor is $b \approx 1$.Combining Eqs. 5 and 6 yields

$\gamma_{1 w}=b \cdot K_{\mathrm{aw}} \cdot e^{-\pi_{\mathrm{M}} A_{\mathrm{D}} / k T}$.

Since the cross-sectional area rather than the molecular weight was shown to be relevant for membrane insertion (Boguslavsky et al. 1994; Fischer et al. 1998) and membrane permeation (Finkelstein 1987), we used the molecular radius $\left(r_{\mathrm{D}}\right)$ derived from the cross-sectional area of the molecule in its membrane-bond conformation $\left(A_{\mathrm{D}}=\pi \cdot r_{\mathrm{D}}{ }^{2}\right)$ instead of the molecular radius $(r)$ approximated from the cube root of the molecular weight (Finkelstein 1987) to calculate the diffusion coefficient of a compound (Eq. 3) ${ }^{1}$.

Combining Eqs. 1, 3, and 7, the permeability coefficient $(P)$ can then be expressed as

$P=\kappa \cdot K_{\mathrm{aw}} \cdot e^{-\pi_{\mathrm{M}}\left(\pi r_{D}^{2}\right) / k T} / r_{D}$,

where $\kappa$ comprises the membrane thickness and the viscosity,

$\kappa=\mathrm{b} \cdot \frac{k T}{6 \pi \eta \Delta x}$.

\footnotetext{
${ }^{1}$ Equation 3 applies in principle to spherical particles. The diffusion coefficient of elongated molecules with a small cross-sectional area may, therefore, be somewhat overestimated.
}

The flux $(\Phi)$ is obtained by combining Eqs. 4 and 8:

$$
\Phi=\kappa \cdot C \cdot K_{\mathrm{aw}} \cdot e^{-\pi_{\mathrm{M}}\left(\pi r_{D}^{2}\right) / k T} / r_{D}
$$

Equation 10 shows that the passive influx across the lipid bilayer depends essentially on the packing density of the membrane $\left(\pi_{\mathrm{M}}\right)$ and on the air-water partition coefficient $\left(K_{\mathrm{aw}}\right)$ and the radius of the drug $\left(r_{\mathrm{D}}\right)$, in the case of electrically neutral compounds (Seelig and Gatlik-Landwojtowicz 2005).

The role of charge for membrane binding and permeation The fraction of the non-ionized form of bases $\left(f_{\mathrm{A}}\right)$ and acids $\left(f_{\mathrm{AH}}\right)$ at a particular $\mathrm{pH}$ that is able to cross the membrane by passive diffusion is calculated as

$f_{\mathrm{A}}=[A] /\left(\left[\mathrm{HA}^{+}\right]+[A]\right)=\left(1+10^{p K_{a}-p H}\right)^{-1}$

and

$f_{\mathrm{AH}}=[\mathrm{AH}] /\left(\left[\mathrm{H}^{+}\right]+\left[\mathrm{A}^{-}\right]\right)=\left(1+10^{\mathrm{p} H-\mathrm{p} K_{\mathrm{a}}}\right)^{-1}$.

The partition coefficient of the permeating species of a base and an acid $\left(\gamma^{*}{ }_{1 \mathrm{w}}\right)$ is

$\gamma_{\mathrm{lw}}^{*}=\gamma_{l \mathrm{w}} \cdot f_{\mathrm{A}}$

and

$\gamma_{\mathrm{lw}}^{*}=\gamma_{l \mathrm{w}} \cdot f_{\mathrm{AH}}$

For many peptides (Beschiaschvili and Seelig 1992), most drugs (Meier et al. 2006) and fatty acids (Cistola et al. 1986), although not all compounds (e.g., tetracaine; Watts and Poile 1986), a $\mathrm{p} K_{\mathrm{a}}$ shift upon membrane binding has been observed. This reflects the fact that the compounds are somewhat less charged after insertion into the lipid bilayer membrane than in aqueous solution. To illustrate, the effect data will be calculated without and with a $\mathrm{p} K_{\mathrm{a}}$ shift $\left(\Delta \mathrm{p} K_{\mathrm{a}}=0.5\right)$. If the partition coefficient, $\gamma_{\mathrm{lw}}$ (Eq. 2) is replaced by $\gamma_{\mathrm{lw}}^{*}$, the permeability coefficient for cationic and anionic compounds $\left(P^{*}\right)$ can then be expressed in analogy to Eq. 8 as

$P^{*}=\kappa \cdot \frac{K_{\mathrm{aw}} \cdot e^{-\pi_{\mathrm{M}}\left(\pi r_{D}^{2}\right) / k T}}{\left(1+10^{\mathrm{p} K_{\mathrm{a}}-\mathrm{pH}}\right)} \cdot \frac{1}{r_{\mathrm{D}}}$

and

$P^{*}=\kappa \cdot \frac{K_{\mathrm{aw}} \cdot e^{-\pi_{\mathrm{M}}\left(\pi r_{D}^{2}\right) / \mathrm{kT}}}{\left(1+10^{\mathrm{pH}-\mathrm{p} K_{\mathrm{a}}}\right)} \cdot \frac{1}{r_{D}}$,

respectively, and the flux $\left(\Phi^{*}\right)$ for cationic and anionic compounds can be given as

$\Phi^{*}=C \cdot \kappa \cdot \frac{K_{\mathrm{aw}} \cdot e^{-\pi_{\mathrm{M}}\left(\pi r_{D}^{2}\right) / k T}}{\left(1+10^{\mathrm{p} K_{\mathrm{a}}-\mathrm{pH}}\right)} \cdot \frac{1}{r_{\mathrm{D}}}$

and

$\Phi^{*}=C \cdot \kappa \cdot \frac{K_{\mathrm{aw}} \cdot e^{-\pi_{\mathrm{M}}\left(\pi r_{D}^{2}\right) / \mathrm{kT}}}{\left(1+10^{\mathrm{pH}-\mathrm{p} K_{\mathrm{a}}}\right)} \cdot \frac{1}{r_{\mathrm{D}}}$,

respectively. For charged molecules, the flux, thus, depends in addition on the ionization constant $\left(\mathrm{p} K_{\mathrm{a}}\right)$ of the compound and the $\mathrm{pH}$ of the environment. 
It has to be noted that the optimal therapeutic drug concentration is often close to the concentration corresponding to the inverse of the air-water partition coefficient $\left(C=1 / K_{\text {aw }}\right.$; Seelig et al. 2000). The passive influx $(\Phi)$ into the cell (Eq. 10) then simplifies to

$\Phi \cong\left(\kappa \cdot e^{-\pi_{\mathrm{m}} A_{\mathrm{D}} / \mathrm{kT}}\right) / r_{\mathrm{D}}$

For uncharged drugs, it depends essentially on the radius $r_{\mathrm{D}}$ of the diffusing molecule (where $A_{\mathrm{D}}=\pi r_{D}{ }^{2}$ ) and the packing density of the membrane $\left(\pi_{\mathrm{M}}\right)$, and for charged drugs, it depends in addition on the $\mathrm{p} K_{\mathrm{a}}$ of the drug and the $\mathrm{pH}$ of the environment (Eqs. 17 and 18).

\section{Measurement of Intrinsic Active Efflux}

Intrinsic transport by Pgp is directly related to the rate of Pgp activity (Gatlik-Landwojtowicz et al. 2006; Litman et al. 1997a), and intrinsic substrates can, therefore, be identified by measuring the ATPase activity of Pgp. Whether one or two ATP molecules are hydrolyzed per transport cycle is still discussed (for review, see Higgins and Linton 2004). In this study, we assumed that one ATP molecule is hydrolyzed (AlShawi et al. 2003). Conventionally, Pgp activity has been measured with inside-out plasma vesicles of MDR1-transfected cells or with reconstituted proteoliposomes by monitoring phosphate release upon ATP hydrolysis (AlShawi et al. 2003; Ambudkar 1998; Litman et al. 1997b, c). We have, for the first time, measured the activity of Pgp in living MDR1-transfected cells as a function of concentration by monitoring the extracellular acidification rate that reveals the rate of ATP synthesis (Gatlik-Landwojtowicz et al. 2004; Gatlik-Landwojtowicz et al. 2006; Landwojtowicz et al. 2002). Pgp activity as a function of drug concentration yields generally bell-shaped activity profiles. They were evaluated using modified Michaelis-Menten kinetics, assuming two binding sites, one for activation and one for inhibition (Litman et al. 1997a). At low-drug concentrations only one binding site is occupied, and binding can be described by a simple Michaelis-Menten kinetics:

$V=V_{0}+\left(V_{\max }-V_{0}\right) C /\left(C+K_{\mathrm{m}}\right)$,

where $V_{0}$ is the basal activity and $V_{\max }$ is the maximum activity; $K_{\mathrm{m}}$, the concentration of half-maximum activation (Michaelis-Menten constant), and $C$, the concentration of the drug in the aqueous environment. If data are derived from extracellular acidification rate (ECAR) measurements (GatlikLandwojtowicz et al. 2006), Eq. 20a is written as follows:

$V=V_{0}+\left(V_{\max }^{\prime}-V_{0}^{\prime}\right) C /\left(C+K_{m}\right)$

where $V_{\max }^{\prime}$ is the maximum activity in living cells overexpressing Pgp and $V_{0}^{\prime}$ the basal cellular activity, including basal Pgp activity and basal cellular activity.
The rate of Pgp activity (log scale) decreases linearly with increasing affinity of the compound to the transporter (or decreasing negative free energy of binding from water to the transporter). The free energy of binding from water to the transporter is the sum of the free energy of membrane partitioning and the free energy of binding of the drug from the lipid membrane to the transporter. The latter is proportional to the number of hydrogen bond acceptor patterns in the drug (Seelig 2006).

\section{Results and Discussion}

Strict Size and Charge Limits for Drugs Observed in Calibration Diagram for BBB Permeation

Injection of amphiphilic drugs into water leads to a spontaneous partitioning between the bulk aqueous phase and the air-water interface. At the air-water interface, drugs organize themselves into a monolayer with the polar head groups remaining in the aqueous environment and the hydrophobic groups pointing toward the air. As the dielectric constant of air $(\varepsilon \approx 1)$ is similar to the dielectric constant of the hydrocarbon region of the lipid membrane $(\varepsilon \approx 2)$, the air-water interface provides an excellent model system for drug orientation at the lipid-water interface. Drugs were, therefore, characterized by measuring the surface pressure as a function of concentration (Gibbs adsorption isotherm). This reveals the air-water partition coefficient $\left(K_{\mathrm{aw}}\right)$, the critical micelle concentration $(\mathrm{CMC})$, and the cross-sectional area $\left(A_{\mathrm{D}}\right)$ of the compound perpendicular to the axis of amphiphilicity (Fischer et al. 1998). The latter corresponds to the cross-sectional area of the membrane-bond conformation (Gerebtzoff and Seelig 2006). These three parameters were used to establish the 3D-calibration plot for BBB permeation (Fischer et al. 1998) displayed in Fig. 2. In this representation, the most hydrophobic compounds are located in the left corner and the most hydrophilic compounds in the upper right corner of the xy-plane of the diagram. Compounds that are known to reach the CNS are shown as open and compounds that are known not to reach the CNS as filled symbols. It is interesting to note that compounds with large crosssectional areas do not reach the CNS even if they are very hydrophobic. Compounds measured previously (Fischer et al. 1998) are indicated as circles, and compounds listed in Table 1 are indicated as squares (Fig. 3). The 12 drugs added to the original calibration diagram show the same cutoff area for $\mathrm{BBB}$ permeation $\left(A_{\mathrm{D}} \geq 70 \AA^{2}\right)$ as the compounds investigated previously (Fischer et al. 1998, Gerebtzoff and Seelig 2006). Diltiazem (12) is a borderline case as will be shown in more detail in Fig. 4. For the sake 


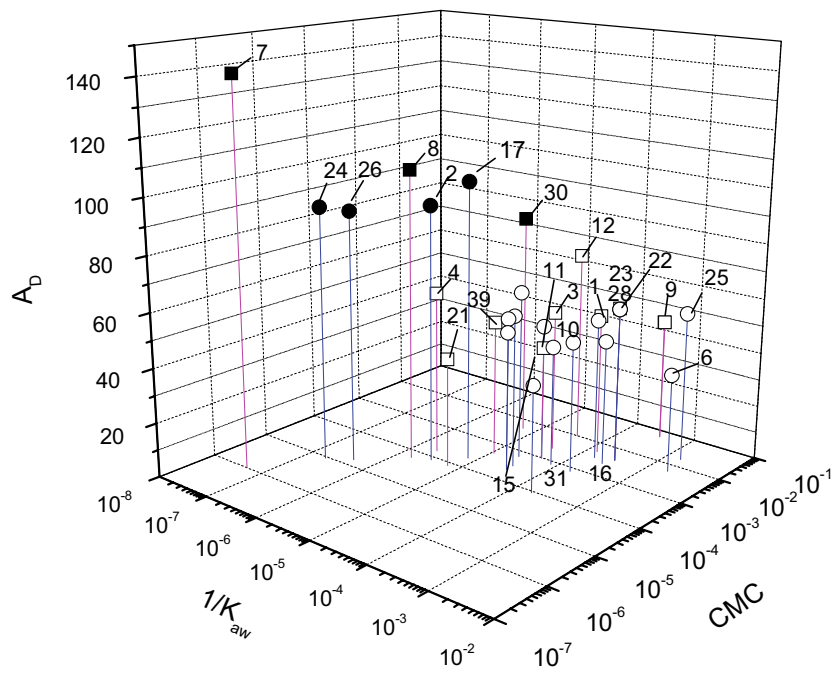

Figure 2 Three-dimensional calibration diagram for BBB permeation for cationic and electrically neutral compounds of clinically wellestablished ability to cross or not to cross the BBB. Compounds that cross the BBB are indicated as open symbols and compounds that do not cross the BBB as solid symbols. The air-water partition coefficient $\left(1 / K_{\text {aw }}\right)$ and the critical micelle concentration $(C M C)$ measured at $\mathrm{pH} 7.4$ are plotted in the $x$ - and $y$-axis, respectively. The $z$-axis shows the cross-sectional area $\left(A_{\mathrm{D}}\right)$ of the drug in its membrane bond conformation (Gerebtzoff and Seelig 2006). $A_{\mathrm{D}}$ values were measured at $\mathrm{pH} 7.4$ and 8.0. To correct for charge repulsion effect values at $\mathrm{pH} 8.0$ are given, unless the area at $\mathrm{pH} 8.0$ was larger than at $\mathrm{pH} 7.4$, which is a sign of drug association and concomitant $\mathrm{p} K_{\mathrm{a}}$ shifts (Gerebtzoff et al. 2004). Data for compounds taken from Fischer et al. (1998) are shown as circles; data for compounds listed in Table 1 are shown as squares: amitriptyline (1), astemizole (2), chlorpromazine (3), cis-flupenthixol (4), clomipramine (5), clonidine (6), cyclosporin A (7), daunorubicin (8), desipramine (9), diazepam (10), dibucaine (11), diltiazem (12), fluoxetine (13), hydroxizine (14), imipramine (15), lidocaine (16), loperamide (17), mequitazine (18), noxiptilip (19), perphenazine (20), progesterone (21), promazine (22), promethazine (23), reserpine (24), tamitinol (25), terfenadine (26), thioridazine (27), trifluoperazine (28), triflupromazine (29), verapamil (30), and zimelidine (31)

of clarity, negatively charged compounds were not included in Fig. 2.

Figure 3 displays the membrane-bound energy-minimized structures of pyrene nonanoic acid and antroyloxystearic acid in comparison to verapamil that is a typical Pgp substrate as determined by molecular modeling (Gerebtzoff and Seelig 2006). The cross-sectional areas of the fatty acids in their amphiphilic membrane-bound conformation clearly differ because of the size and the position of the fluorescence labels. Their calculated $\mathrm{p} K_{\mathrm{a}}$ values also differ as seen in Table 1 due to the different inductive effects and the different positions of the fluorescence labels.

Passive Influx Depends on the Size and the Charge of the Molecule

Figure $4 \mathrm{a}$ and $\mathrm{b}$ displays passive influx $\left(\Phi^{*}\right)$ as a function of the cross-sectional area $\left(A_{\mathrm{D}}\right)$ for the 12 compounds listed in
Table 1. Passive influx $\left(\Phi^{*}\right)$ into the cell was calculated for the concentration of half-maximum Pgp activity for each compound according to Eq. 17 for the drugs and Eq. 18 for the fatty acids. The charge of many drugs and fatty acids is reduced upon membrane insertion (see "Material and Methods"). To illustrate this effect, we calculated passive influx without (Fig. 4a) and with (Fig. 4b) a $\mathrm{p} K_{\mathrm{a}}$ shift. The further parameters used for the calculation of passive influx are given in the legend to Fig. 4. For compounds with small cross-sectional areas, e.g., the electrically neutral progesterone (9), influx is orders of magnitude higher than for

Table 1 The ionization constant $\left(\mathrm{p} K_{\mathrm{a}}\right)$, the air-water partition coefficient $\left(K_{\mathrm{aw}}\right)$, the cross-sectional area $\left(A_{\mathrm{D}}\right)$, the concentration of half-maximum activation of Pgp $\left(K_{1}\right)$, and the maximum activity $\left(V_{1}\right)$ of compounds 1-12 are measured values taken from GatlikLandwojtowicz et al. (2006). The ionization constant ( $\left.\mathrm{p} K_{\mathrm{a}}\right)$ and the cross-sectional area $\left(A_{\mathrm{D}}\right)$ of the fatty acid analogs $13-15$ are calculated values as described in Gerebtzoff and Seelig (2006)

\begin{tabular}{|c|c|c|c|c|c|c|}
\hline Number & Compound & $\mathrm{p} K_{\mathrm{a}}$ & $\begin{array}{l}K_{\mathrm{aw}} \\
{\left[\mathrm{mM}^{-1}\right]} \\
\mathrm{pH} 7.4\end{array}$ & $\begin{array}{l}A_{\mathrm{D}}\left[\AA^{2}\right], \\
\mathrm{pH} 8.0\end{array}$ & $\begin{array}{l}K_{1} \\
{[\mu \mathrm{M}]}\end{array}$ & $\begin{array}{l}V_{1} \\
\text { [fold] }\end{array}$ \\
\hline 1 & Amitriptyline & 9.4 & 7.1 & 52 & $\begin{array}{c}6.4 \pm \\
0.2\end{array}$ & $\begin{array}{r}1.9 \pm \\
0.01\end{array}$ \\
\hline 2 & Chlorpromazine & 9.2 & 21.5 & 42 & $\begin{array}{c}5.7 \pm \\
0.0\end{array}$ & $\begin{array}{c}2.0 \pm \\
0.3\end{array}$ \\
\hline 3 & Cis-Flupenthixol & 7.8 & 453 & 60 & $\begin{array}{c}3.0 \pm \\
1.8\end{array}$ & $\begin{array}{c}1.4 \pm \\
0.2\end{array}$ \\
\hline 4 & Cyclosporin A & - & $1,9952.6$ & 140 & $\begin{array}{c}0.02 \pm \\
0.01\end{array}$ & $\begin{array}{c}1.1 \pm \\
0.1\end{array}$ \\
\hline 5 & Daunorubicin & 8.4 & 588.8 & 106 & $\begin{array}{c}0.6 \pm \\
0.3\end{array}$ & $\begin{array}{r}1.1 \pm \\
0.05\end{array}$ \\
\hline 6 & Dibucaine & 8.5 & 11.3 & 52 & $\begin{array}{c}10.9 \pm \\
1.0\end{array}$ & $\begin{array}{r}2.0 \pm \\
0.2\end{array}$ \\
\hline 7 & Diltiazem & 8.9 & 26.9 & 70 & $\begin{array}{r}3.5 \pm \\
0.4\end{array}$ & $\begin{array}{c}1.8 \pm \\
0.2\end{array}$ \\
\hline 8 & Lidocaine & 7.6 & 2.9 & 45 & $\begin{array}{c}375.6 \pm \\
91.3\end{array}$ & $\begin{array}{c}1.5 \pm \\
0.1\end{array}$ \\
\hline 9 & Progesterone & - & 157 & 40 & $\begin{array}{c}5.0 \pm \\
0.7\end{array}$ & $\begin{array}{c}1.7 \pm \\
0.2\end{array}$ \\
\hline 10 & Trifluoperazine & 8.09 & 342 & 57.4 & $\begin{array}{c}3.6 \pm \\
0.0\end{array}$ & $\begin{array}{c}1.6 \pm \\
0.1\end{array}$ \\
\hline 11 & Triflupromazine & 9.1 & 91.1 & 50 & $\begin{array}{c}2.0 \pm \\
0.5\end{array}$ & $\begin{array}{c}1.7 \pm \\
0.1\end{array}$ \\
\hline 12 & $(\mathrm{R} / \mathrm{S})$-Verapamil & 8.92 & 166.0 & 82 & $\begin{array}{c}0.5 \pm \\
0.1\end{array}$ & $\begin{array}{c}1.6 \pm \\
0.1\end{array}$ \\
\hline 13 & Palmitic acid & 4.96 & 5000 & 27 & 0.2 & ND \\
\hline 14 & $\begin{array}{l}\text { Pyrene nonanoic } \\
\text { acid }\end{array}$ & 4.78 & 6000 & 43 & 0.2 & ND \\
\hline 15 & $\begin{array}{l}\text { Antroyloxystearic } \\
\text { acid }\end{array}$ & 2.84 & 7000 & 73 & 0.2 & ND \\
\hline
\end{tabular}

The air-water partition coefficient $\left(K_{\mathrm{aw}}\right)$ for palmitic acid was measured (X. Li-Blatter, unpublished results). The values of the two analogs were estimated. The concentration of half-maximum activation of the hypothetical efflux transporter (MRP1) was estimated according to $K_{\mathrm{aw}} \cdot K_{\mathrm{m}} \approx 1$ (see Seelig and Landwojtowicz 2000). The rate of transport was assumed to be similar to Pgp. 

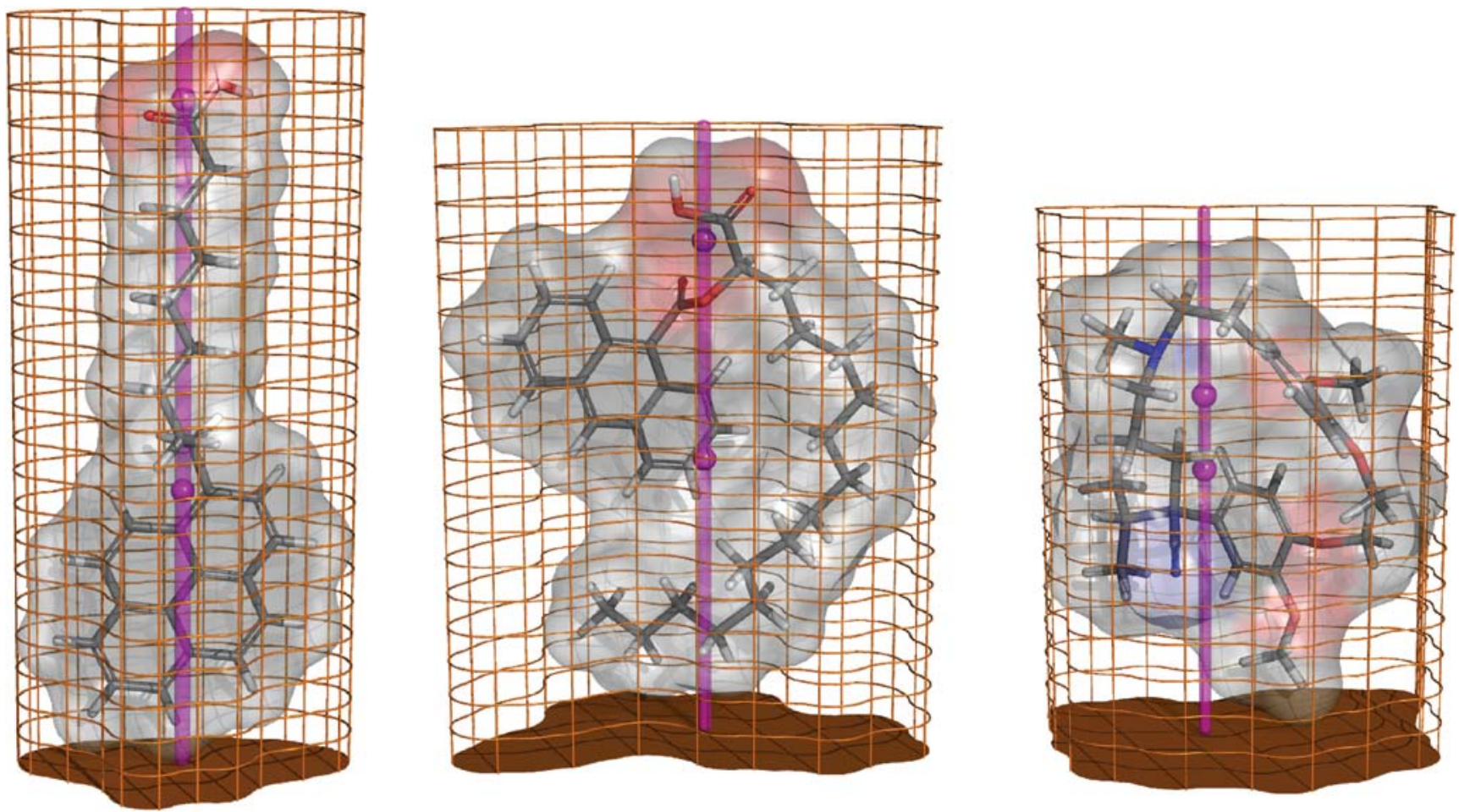

Figure 3 Pyrene nonanoic acid (14), antroyloxystearic acid (15), and verapamil (12) (for number see Table 1). The molecules are shown in their membrane-bound amphiphilic conformation as described in Gerebtzoff and Seelig (2006)

compounds with large cross-sectional areas, e.g., the electrically neutral cyclosporin A (4). The influence of the $\mathrm{p} K_{\mathrm{a}}$ value is seen, e.g., by the comparison of amitriptyline (1) and dibucaine (6), which show the same cross-sectional area. Amitriptyline, which has a higher $\mathrm{p} K_{\mathrm{a}}$ value than dibucaine, is much more at risk to be exported than dibucaine.

Due to their different cross-sectional areas and $\mathrm{p} K_{\mathrm{a}}$ values, the three fatty acids show distinctly different passive influx rates as shown in Fig. 4. The uncharged fraction of molecules exhibiting small cross-sectional areas, e.g., unlabelled fatty acids, cross the membrane rapidly (for review, see (Hamilton 1998; Hamilton and Kamp 1999), whereas fatty acids labeled with fluorescent groups exhibiting larger cross-sectional areas show a lower flip-flop rate. The calculated passive influx of fatty acids (Fig. 3) correlates well with measured flip-flop rates.

Active Efflux Depends on the Binding Affinity of the Drug to the Transporter

Active efflux was measured in living MDR1 mouse embryo fibroblasts (Gatlik-Landwojtowicz et al. 2006) and in the corresponding inside-out membrane vesicles (Aanismaa and Seelig 2007). The concentrations of half-maximum activity were identical in the two systems. However, the rate of intrinsic transport (or Pgp activity) was higher in living cells than in inside-out plasma membrane vesicles (Aanismaa and Seelig 2007). Active efflux $(-V)$ as a function of the cross- sectional area $\left(A_{\mathrm{D}}\right)$ was included in Fig. 4. Active efflux $(-V)$ was calculated according to Eq. 20 using the maximum fold activity, $V_{1}$ or $\left(V_{\max }\right)$ measured in MDR1-transfected mouse embryo fibroblasts (Gatlik-Landwojtowicz et al. 2006; see Table 1). MRP activation as a function of concentrations has not yet been measured to our knowledge for the fatty acids given in Table 1. Because MRP and Pgp function in a similar manner, it is reasonable to assume similar turnover numbers for the two proteins.

Recent data (Aanismaa and Seelig 2007) show that the rate of active efflux decreases exponentially with increasing binding affinity of the drug from water to the transporter. The binding affinity of the drug from water to the transporter is the sum of the binding affinity of the drug from water to the lipid membrane and the binding affinity from the lipid membrane to the transporter (Gatlik-Landwojtowicz et al. 2006). The binding affinity from the lipid phase to the transporter increases with the number and strength of hydrogen bond acceptor groups that seem to interact with numerous hydrogen bond donor groups in the transmembrane domains of the transporter (Omote and Al-Shawi 2006; Seelig et al. 2000). For the present set of compounds, the number of hydrogen bond acceptor groups per drug increases with the cross-sectional area of the drug (Gatlik-Landwojtowicz et al. 2006; Seelig and Gatlik-Landwojtowicz 2005). The plot of active efflux $(-V)$ (log scale) as a function of the crosssectional area $\left(A_{\mathrm{D}}\right)$ is, therefore, approximately linear as seen in Fig. 4 (stars). In contrast to passive influx that decreases 

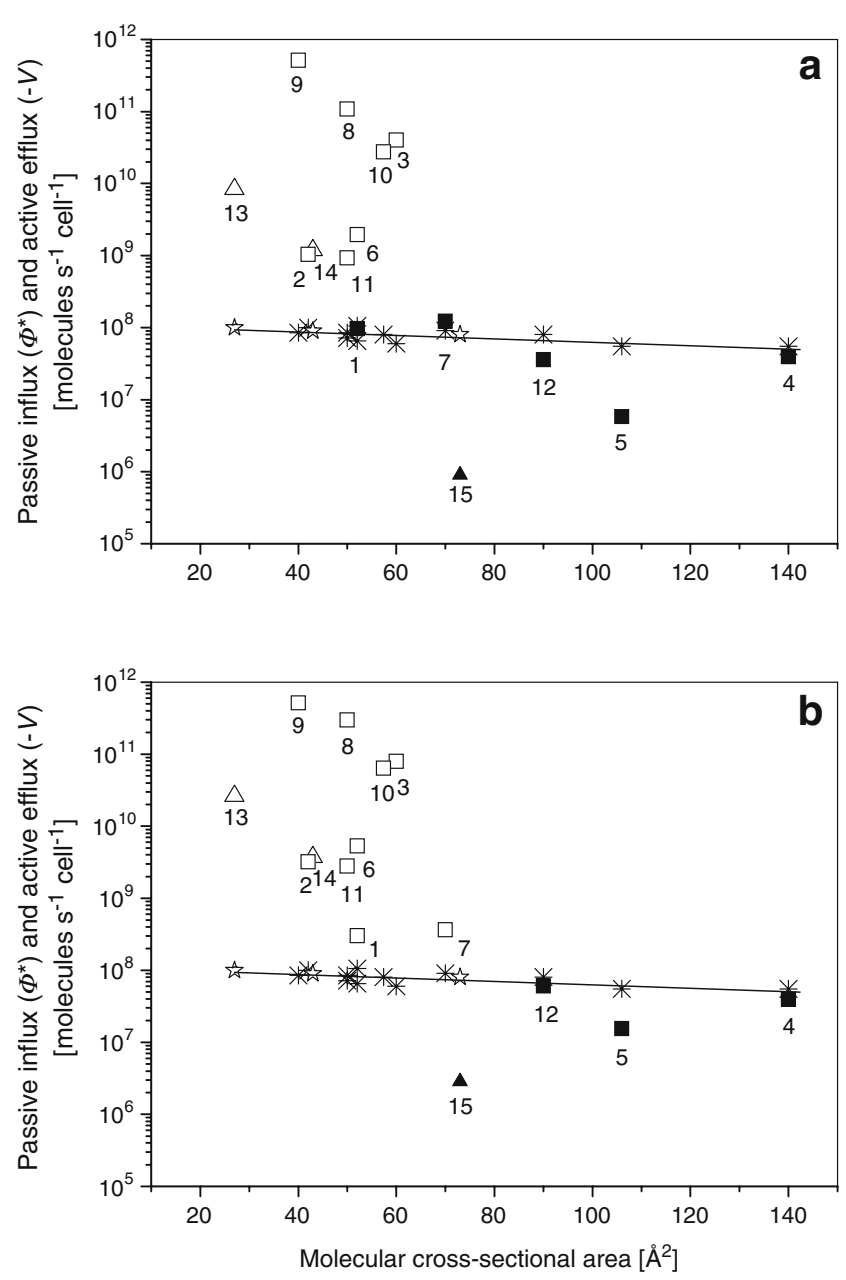

Figure 4 a, b Passive influx ( $\left.\Phi^{*}\right)$ of drugs (filled and open circles) and fatty acids (filled and open triangles) and active efflux $(-V)$ of drugs (asterisk) by Pgp and of fatty acids (open stars) by MRPs plotted as a function of the molecular cross-sectional area $\left(A_{\mathrm{D}}\right)$. a Influx was calculated taking into account no $\mathrm{p} K_{\mathrm{a}}$ shift upon drug binding to the lipid membrane. b Influx was calculated taking into account a $\mathrm{p} K_{\mathrm{a}}$ shift $\left(\Delta \mathrm{p} K_{\mathrm{a}}=0.5\right)$ that reduces the charge of all compounds upon membrane binding. Compounds with high influx $\left(\Phi^{*}>10^{8}\right.$ molecules $\mathrm{s}^{-1}$ cell $\left.^{-1}\right)$ are represented as open symbols and compounds with low influx $\left(\Phi^{*}<10^{8}\right.$ molecules $\mathrm{s}^{-1}$ cell $\left.^{-1}\right)$ as filled symbols. The latter are apparent substrate for efflux transporters (generally called "substrates"). The concentration chosen for these calculations was the concentration of half-maximum transporter activity $\left(K_{1}\right)$. It should be noted that the product of the air-water partition coefficient $\left(K_{\mathrm{aw}}\right)$ and the concentration of half-maximum activation $\left(K_{\mathrm{m}}\right)$ is close to one $\left(K_{\mathrm{aw}} K_{\mathrm{m}} \approx 1\right)$ (Seelig and Landwojtowicz 2000). The drug concentration $\left(C=1 / K_{\mathrm{aw}}\right)$, thus, corresponds approximately to the concentration of half-maximum transporter activation $\left(K_{1}\right)$. Further parameters used are the thickness, $\Delta x=50 \AA$; the viscosity, $\eta=1 \mathrm{P}$; and the packing density $\pi_{\mathrm{M}}=35 \mathrm{mN} / \mathrm{m}$ (Seelig and Gerebtzoff 2006) of the membrane and the lipid membrane surface area per cell $A_{\text {lip }}=2.26 \cdot 10^{-7} \mathrm{~cm}^{2}$. Compounds are numbered as in Table 1

with increasing cross-sectional area and charge, neither the cross-sectional area (Seelig and Gatlik-Landwojtowicz 2005) nor charge (e.g., Omote and Al-Shawi 2002) hinder active efflux by Pgp and MRP, provided the molecules can reach the inner leaflet of the lipid membrane. This suggests that the efflux pathway through the protein is significantly more flexible and polar than the influx pathway across the lipid bilayer membrane.

Apparent or Net Flux is the Sum of Passive Influx and Active Efflux

Apparent or net transport $(J)$ across a cell membrane can be estimated as the sum of passive influx $(\Phi)$ (or $\Phi^{*}$ ) and active efflux $(-V)$ :

$J=\Phi-V$.

According to Eq. 21, apparent export is only observed if the value of active efflux $(-V)$ is higher than the value of passive influx $(\Phi)$ or $\left(\Phi^{*}\right)$. The comparison of passive influx $\left(\Phi^{*}\right)$ and active efflux ( $-V$ (both given in the same units) is seen in Fig. $4 \mathrm{a}$ and b. Compounds with high influx $\left(\Phi^{*} \gg 10^{8}\right.$ molecules $\mathrm{s}^{-1}$ cell $^{-1}$ ) are represented as open symbols and compounds with low influx $\left(\Phi^{*} \ll 10^{8}\right.$ molecules s ${ }^{-1}$ cell $\left.^{-1}\right)$ as filled symbols. It is evident that compounds with low charge and/or small cross-sectional area $\left(A_{\mathrm{D}}<70 \AA^{2}\right)$ generally exhibit significantly higher passive influx $\left(\Phi^{*}\right)$ than active efflux $(-V)$, whereas large or highly charged compounds generally exhibit lower influx than efflux. The compounds, chlorpromazine, cis-flupenthixol, dibucaine, lidocaine, progesterone, trifluoperazine, and triflupromazine, thus, cross the membrane barrier despite being intrinsic Pgp substrates, whereas cyclosporin A, daunorubicin, and verapamil are clear apparent substrates that do not reach the CNS in significant concentrations. Amitriptyline and diltiazem are borderline compounds. Without taking into account a $\mathrm{p} K_{\mathrm{a}}$ shift (Fig. 4a), they are predicted to be apparent Pgp substrates; if a $\mathrm{p} K_{\mathrm{a}}$ shift is taken into account, they seem to reach the CNS at low concentrations (Fig. 4b). The present simple prediction of apparent substrates perfectly agrees with biological results at the BBB (for amitriptyline and cyclosporin A, Thuerauf and Fromm 2006; for daunorubicin, Zhao et al. 2002; and for diltiazem and verapamil, Pani et al. 1990). Not only the twelve drugs but also the three fatty acids show distinctly different passive influx rates due to their different crosssectional areas and different $\mathrm{p} K_{\mathrm{a}}$ values. The two slimmer molecules are likely to reach the CNS by passive diffusion despite eventually being intrinsic substrates of MRPs, whereas antroyloxystearic acid is likely to be an apparent substrate and is, therefore, unlikely to reach the CNS.

The strict area and charge limitations observed in BBB and IB calibration diagrams (Fischer et al. 1998; Fischer et al. 1998; Gerebtzoff and Seelig 2006) can be explained on the basis of Fig. 4, which reveals that if a certain charge and size limit is reached, influx $(\Phi)$ becomes lower than efflux $(-\mathrm{V})$. If the membrane exhibits a lower lateral packing density as, e.g., the IB, the limiting cross-sectional area is larger (Fischer et al.1998). 


\section{Conclusions}

Three-dimensional calibration diagram for BBB permeation established previously using the cross-sectional area $\left(A_{\mathrm{D}}\right)$, the air-water partition coefficient $\left(K_{\mathrm{aw}}\right)$, and the critical micelle concentration $\left(C M C_{\mathrm{D}}\right)$ as parameters (Fischer et al. 1998; Gerebtzoff and Seelig 2006; Seelig et al. 1994) revealed a limiting cross-sectional area $\left(A_{\mathrm{D}}=70 \quad \AA^{2}\right)$. In addition a limiting ionization constant $\left(\mathrm{p} K_{\mathrm{a}} \leq 4\right.$ for acids and $\mathrm{p} K_{\mathrm{a}} \geq 10$ for bases) was observed. Incorporation of additional compounds into the original $3 \mathrm{D}$ calibration diagram corroborated the limiting values for $\mathrm{BBB}$ permeation. To rationalize the strict area and charge limitation at the BBB, we investigated the relationship between passive influx $(\Phi)$ and active efflux by $\mathrm{ABC}$ transporters and determined net influx $(J)$ as the sum of the two. Passive influx $(\Phi)$ was calculated on the basis of Stokesian diffusion, taking into account the airwater partition, the cross-sectional area of the membranebound conformation, and the ionization constant of the compound, as well as the lateral packing density of the membrane. Active efflux across the BBB was measured in MDR1-transfected mouse embryo fibroblasts, which reveal a Pgp expression level comparable to that of the BBB. Active efflux depends on the affinity of the substrate to the transporter, which in turn, correlates with the number and strength of hydrogen bond interactions between the substrate and the transporter. While passive influx $(\Phi)$ changes by several orders of magnitude with the size and the charge of the molecule, active efflux $(-V)$ changes by less than one order of magnitude for a given cell line. As long as molecules are small (and influx is high), active transport remains furtive. It becomes apparent only if molecules exhibit a large cross-sectional area or a high charge and efflux $(-V)$ start to be larger than influx $(\Phi)$. The crosssectional area $\left(A_{\mathrm{D}}\right)$ and the charge $\left(\mathrm{p} K_{\mathrm{a}}\right)$, therefore, dominate the net flux $(J)$ of drugs across the BBB. Whether a compound is a substrate for the efflux transporters Pgp and MRP1, however, not only depends on the characteristics of the compound but also on the packing density of the lipid membrane and the expression level of the transporters.

Acknowledgment I am grateful to Dr. Grégori Gerebtzoff for modeling the compounds in Fig. 3 and to Dr. Renate Reiter and Dr. Andreas Zumbühl for carefully reading the ms. This work was supported by the Swiss National Science Foundation, grant no. 3100-107793.

\section{References}

Aanismaa, P., \& Seelig, A. (2007). P-glycoprotein kinetics measured in plasma membrane vesicles and living cells. Biochemistry, 46, 3394-3404.

Al-Shawi, M. K., Polar, M. K., Omote, H., \& Figler, R. A. (2003). Transition state analysis of the coupling of drug transport to ATP hydrolysis by P-glycoprotein. Journal of Biological Chemistry, 278, 52629-52640.

Altenbach, C., \& Seelig, J. (1985). Binding of the lipophilic cation tetraphenylphosphonium to phosphatidylcholine membranes. Biochimica Et Biophysica Acta, 818, 410.

Ambudkar, S. V. (1998). Drug-stimulatable ATPase activity in crude membranes of human MDR1-transfected mammalian cells. Methods in Enzymology, 292, 504-514.

Bassolino-Klimas, D., Alper, H. E., \& Stouch, T. R. (1993). Solute diffusion in lipid bilayer membranes: An atomic level study by molecular dynamics simulation. Biochemistry, 32, 12624-12637.

Begley, D. J. (2004). ABC transporters and the blood-brain barrier. Current Pharmaceutical Design, 10, 1295-1312.

Beschiaschvili, G., \& Seelig, J. (1992). Peptide binding to lipid bilayers. Nonclassical hydrophobic effect and membrane-induced pK shifts. Biochemistry, 31, 10044-10053.

Boguslavsky, V., Rebecchi, M., Morris, A. J., Jhon, D. Y., Rhee, S. G., \& McLaughlin, S. (1994). Effect of monolayer surface pressure on the activities of phosphoinositide-specific phospholipase Cbeta 1, -gamma 1, and -delta 1. Biochemistry, 33, 3032-3037.

Burton, P. S., Goodwin, J. T., Vidmar, T. J., \& Amore, B. M. (2002). Predicting drug absorption: How nature made it a difficult problem. Journal of Pharmacology and Experimental Therapeutics, 303, 889-895.

Cistola, D. P., Atkinson, D., Hamilton, J. A., \& Small, D. M. (1986). Phase behavior and bilayer properties of fatty acids: Hydrated 1:1 acid-soaps. Biochemistry, 25, 2804-2812.

Didziapetris, R., Japertas, P., Avdeef, A., \& Petrauskas, A. (2003). Classification analysis of $P$-glycoprotein substrate specificity. Journal of Drug Targeting, 11, 391-406.

Finkelstein, A. (1987). Water movement through lipid bilayers, pores, and plasma membranes. Theory and reality. New York: Wiley.

Fischer, H., Gottschlich, R., \& Seelig, A. (1998). Blood-brain barrier permeation: molecular parameters governing passive diffusion. Journal of Membrane Biology, 165, 201-211.

Gatlik-Landwojtowicz, E., Aanismaa, P., \& Seelig, A. (2004). The rate of $P$-glycoprotein activation depends on the metabolic state of the cell. Biochemistry, 43, 14840-14851.

Gatlik-Landwojtowicz, E., Aanismaa, P., \& Seelig, A. (2006). Quantification and characterization of $P$-glycoprotein-substrate interactions. Biochemistry, 45, 3020-3032.

Gerebtzoff, G., Li-Blatter, X., Fischer, H., Frentzel, A., \& Seelig, A. (2004). Halogenation of drugs enhances membrane binding and permeation. ChemBioChem, 5, 676-684.

Gerebtzoff, G., \& Seelig, A. (2006). In silico prediction of bloodbrain barrier permeation using the calculated molecular crosssectional area as main parameter. Journal of Chemical Information and Modeling, 46, 2638-2650.

Golin, J., Ambudkar, S. V., Gottesman, M. M., Habib, A. D., Sczepanski, J., Ziccardi, W., et al. (2003). Studies with novel Pdr5p substrates demonstrate a strong size dependence for xenobiotic efflux. Journal of Biological Chemistry, 278, 5963-5969.

Hamilton, J. A. (1998). Fatty acid transport: Difficult or easy? Journal of Lipid Research, 39, 467-481.

Hamilton, J. A., \& Kamp, F. (1999). How are free fatty acids transported in membranes? Is it by proteins or by free diffusion through the lipids? Diabetes, 48, 2255-2269.

Higgins, C. F., \& Linton, K. J. (2004). The ATP switch model for ABC transporters. Natural Structural Molecular Biology, 11, 918-926.

Kamp, F., \& Hamilton, J. A. (1992). pH gradients across phospholipid membranes caused by fast flip-flop of un-ionized fatty acids. Proceedings of the National Academy of Sciences of the United States of America, 89, 11367-11370.

Landwojtowicz, E., Nervi, P., \& Seelig, A. (2002). Real-time monitoring of P-glycoprotein activation in living cells. Biochemistry, 41, $8050-8057$. 
Litman, T., Nielsen, D., Skovsgaard, T., Zeuthen, T., \& Stein, W. D. (1997a). ATPase activity of $P$-glycoprotein related to emergence of drug resistance in Ehrlich ascites tumor cell lines. Biochimica Et Biophysica Acta, 1361, 147-158.

Litman, T., Zeuthen, T., Skovsgaard, T., \& Stein, W. D. (1997b). Competitive, non-competitive and cooperative interactions between substrates of P-glycoprotein as measured by its ATPase activity. Biochimica Et Biophysica Acta, 1361, 169-176.

Litman, T., Zeuthen, T., Skovsgaard, T., \& Stein, W. D. (1997c). Structure-activity relationships of P-glycoprotein interacting drugs: Kinetic characterization of their effects on ATPase activity. Biochimica Et Biophysica Acta, 1361, 159-168.

Mealey, K. L., Bentjen, S. A., Gay, J. M., \& Cantor, G. H. (2001). Ivermectin sensitivity in collies is associated with a deletion mutation of the mdr1 gene. Pharmacogenetics, 11, 727-733.

Meier, M., Blatter, X. L., Seelig, A., \& Seelig, J. (2006). Interaction of verapamil with lipid membranes and $P$-glycoprotein: connecting thermodynamics and membrane structure with functional activity. Biophysical Journal, 91, 2943-2955.

Ogawa, K., Yumoto, R., Hamada, N., Nagai, J., \& Takano, M. (2006). Interaction of valproic acid and carbapenem antibiotics with multidrug resistance-associated proteins in rat erythrocyte membranes. Epilepsy Research, 71, 76-87.

Omote, H., \& Al-Shawi, M. K. (2002). A novel electron paramagnetic resonance approach to determine the mechanism of drug transport by P-glycoprotein. Journal of Biological Chemistry, 277, 45688-45694.

Omote, H., \& Al-Shawi, M. K. (2006). Interaction of transported drugs with the lipid bilayer and $P$-glycoprotein through a solvation exchange mechanism. Biophysical Journal, 90, 4046-4059.

Pani, L., Kuzmin, A., Diana, M., De Montis, G., Gessa, G. L., \& Rossetti, Z. L. (1990). Calcium receptor antagonists modify cocaine effects in the central nervous system differently. European Journal of Pharmacology, 190, 217-221.

Pauletti, G. M., Okumu, F. W., \& Borchardt, R. T. (1997). Effect of size and charge on the passive diffusion of peptides across Caco2 cell monolayers via the paracellular pathway. Pharmaceutical Research, 14, 164-168.

Saparov, S. M., Antonenko, Y. N., \& Pohl, P. (2006). A new model of weak acid permeation through membranes revisited: Does Overton still rule? Biophysical Journal, 90, L86-L88.

Schinkel, A. H., Wagenaar, E., van Deemter, L., Mol, C. A., \& Borst, P. (1995). Absence of the mdrla P-Glycoprotein in mice affects tissue distribution and pharmacokinetics of dexamethasone, digoxin, and cyclosporin A. Journal of Clinical Investigation, 96, 1698-1705.
Seelig, A. (1987). Local anesthetics and pressure: A comparison of dibucaine binding to lipid monolayers and bilayers. Biochimica Et Biophysica Acta, 899, 196-204.

Seelig, A. (1998). A general pattern for substrate recognition by $P$ glycoprotein. European Journal of Biochemistry, 251, 252-261.

Seelig, A. (2006). Unraveling membrane-mediated substrate-transporter interactions. Biophysical Journal, 90, 3825-3826.

Seelig, A., Blatter, X. L., \& Wohnsland, F. (2000). Substrate recognition by $\mathrm{P}$-glycoprotein and the multidrug resistanceassociated protein MRP1: A comparison. International Journal of Clinical Physical Therapy, 38, 111-121.

Seelig, A., \& Gatlik-Landwojtowicz, E. (2005). Inhibitors of multidrug efflux transporters: Their membrane and protein interactions. Mini Reviews in Medicinal Chemistry, 5, 135-151.

Seelig, A., \& Gerebtzoff, G. (2006). Enhancement of drug absorption by noncharged detergents through membrane and P-glycoprotein binding. Expert Opinion on Drug Metabolism \& Toxicology, 2, $733-752$.

Seelig, A., Gottschlich, R., \& Devant, R. M. (1994). A method to determine the ability of drugs to diffuse through the blood-brain barrier. Proceedings of the National Academy of Sciences of the United States of America, 91, 68-72.

Seelig, A., \& Landwojtowicz, E. (2000). Structure-activity relationship of $P$-glycoprotein substrates and modifiers. European Journal of Pharmaceutical Sciences, 12, 31-40.

Seelig, A., \& Seelig, J. (2002). Membrane structure, in encyclopedia of physical science and technology (9th ed., pp. 355-367). New York: Academic.

Seelig, J., \& Seelig, A. (1980). Lipid conformation in model membranes and biological membranes. Quarterly Reviews of Biophysics, 13, 19-61.

Thuerauf, N., \& Fromm, M. F. (2006). The role of the transporter $P$ glycoprotein for disposition and effects of centrally acting drugs and for the pathogenesis of CNS diseases. European Archives of Psychiatry and Clinical Neuroscience, 256, 281-286.

Varma, M. V., Sateesh, K., \& Panchagnula, R. (2005). Functional role of $P$-glycoprotein in limiting intestinal absorption of drugs: Contribution of passive permeability to P-glycoprotein mediated efflux transport. Molecular Pharmacology, 2, 12-21.

Watts, A., \& Poile, T. W. (1986). Direct determination by 2 H-NMR of the ionization state of phospholipids and of a local anaesthetic at the membrane surface. Biochimica Et Biophysica Acta, 861, 368-372.

Zhao, Y. L., Du, J., Kanazawa, H., Sugawara, A., Takagi, K., Kitaichi, K., et al. (2002). Effect of endotoxin on doxorubicin transport across blood-brain barrier and $P$-glycoprotein function in mice. European Journal of Pharmacology, 445, 115-123. 